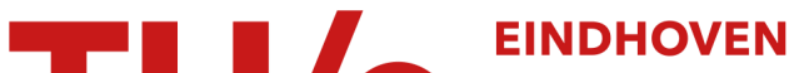 UNIVERSITY OF TECHNOLOGY
}

\section{The relevance of non-generic events in scale space models}

Citation for published version (APA):

Kuijper, A., \& Florack, L. M. J. (2004). The relevance of non-generic events in scale space models. International Journal of Computer Vision, 57(1), 67-84. https://doi.org/10.1023/B:VISI.0000013091.14851.24

DOI:

10.1023/B:VISI.0000013091.14851.24

Document status and date:

Published: 01/01/2004

\section{Document Version:}

Publisher's PDF, also known as Version of Record (includes final page, issue and volume numbers)

\section{Please check the document version of this publication:}

- A submitted manuscript is the version of the article upon submission and before peer-review. There can be important differences between the submitted version and the official published version of record. People interested in the research are advised to contact the author for the final version of the publication, or visit the $\mathrm{DOI}$ to the publisher's website.

- The final author version and the galley proof are versions of the publication after peer review.

- The final published version features the final layout of the paper including the volume, issue and page numbers.

Link to publication

\section{General rights}

Copyright and moral rights for the publications made accessible in the public portal are retained by the authors and/or other copyright owners and it is a condition of accessing publications that users recognise and abide by the legal requirements associated with these rights.

- Users may download and print one copy of any publication from the public portal for the purpose of private study or research.

- You may not further distribute the material or use it for any profit-making activity or commercial gain

- You may freely distribute the URL identifying the publication in the public portal.

If the publication is distributed under the terms of Article 25fa of the Dutch Copyright Act, indicated by the "Taverne" license above, please follow below link for the End User Agreement:

www.tue.nl/taverne

Take down policy

If you believe that this document breaches copyright please contact us at:

openaccess@tue.nl

providing details and we will investigate your claim. 
International Journal of Computer Vision 57(1), 67-84, 2004 (c) 2004 Kluwer Academic Publishers. Manufactured in The Netherlands.

\title{
The Relevance of Non-Generic Events in Scale Space Models
}

\author{
ARJAN KUIJPER \\ IT University of Copenhagen, Department of Innovation, Glentevej 67, DK-2400 Copenhagen, Denmark \\ arjan@itu.dk \\ LUC M.J. FLORACK \\ Technical University Eindhoven, Department of Biomedical Engineering, Den Dolech 2, \\ NL-5600 MB Eindhoven, The Netherlands \\ 1.m.j.florack@tue.nl
}

Received January 18, 2002; Revised April 30, 2003; Accepted May 2, 2003

\begin{abstract}
In order to investigate the deep structure of Gaussian scale space images, one needs to understand the behaviour of spatial critical points under the influence of blurring. We show how the mathematical framework of catastrophe theory can be used to describe and model the behaviour of critical point trajectories when various different types of generic events, viz. annihilations and creations of pairs of spatial critical points, (almost) coincide. Although such events are non-generic in mathematical sense, they are not unlikely to be encountered in practice due to numerical limitations. Furthermore, the behaviour of these trajectories leads to the observation that fine-to-coarse tracking of critical points doesn't suffice, since they can form closed loops in scale space. The modelling of the trajectories include these loops. We apply the theory to an artificial image and a simulated MR image and show the occurrence of the described behaviour.
\end{abstract}

Keywords: scale space, catastrophe theory, critical points, topology, deep structure, multi-scale segmentation

\section{Introduction}

The presence of structures of various sizes in an image requires image analysis tools capable of dealing with multiple levels of resolution. Various multi-scale paradigms have been developed (Salden, 1996), giving rise to several new topics of interest. For instance, can specific properties at some scale be related to similar properties at other scales? And if so, what can be said about the way such properties change over scale.

\subsection{Scale Space History}

The concept of Gaussian, or linear scale space has been introduced in the English image literature by Witkin (1983) and Koenderink (1984). They showed that the natural way to represent an image at finite resolution is by convolving it with a Gaussian, thus obtaining a smoothened image at a scale determined by the bandwidth. It has been shown by Weickert et al. (1997, 1999), that the concept of scale space was introduced in Japan about twenty years earlier (Iijima, 1962; Otsu, 1981). Due to the fact that these papers were in Japanese, they remained unnoticed in the Western world. In their papers Weickert et al. give an overview of the several axioms leading to the paradigm of linear scale space. This approach has led to the formulation of various invariant expressions-expressions that are independent of the coordinates-that capture certain features in an image at distinct levels of scale (Duncan and Ayache, 2000; Florack, 1997; Florack et al., 1992, 1993, 1994a, 1994b, 1996; Kalitzin et al., 2001). Such invariant features are potential candidates 
that one would like to trace over scale and the topological changes of which one would like to investigate, e.g. splitting, merging, creation or vanishing. Nowadays, (properties of) scale spaces are widely used in image analysis, segmentation, clustering and statistical analysis (Johansen, 1996; ter Haar Romeny et al., 1997; Nielsen et al., 1999; Kerckhove, 2001). Introductions to scale space can be found in several books (Florack, 1997; Lindeberg, 1994; Sporring et al., 1997).

\subsection{Deep Structure}

In this paper we focus on linear, Gaussian, scale space. This has the advantage that each scale level only requires the choice of an appropriate scale and that the image intensity at that level follows linearly from any previous level. It is therefore possible to trace the evolution of certain image entities over scale. The exploitation of various scales simultaneously has been referred to as deep structure by Koenderink (1984). It pertains to the dynamic change of the image from highly detailedincluding noise - to highly smoothened. Furthermore, it may be expected that large structures "live" longer than small structures (a reason that Gaussian blur is used to suppress noise). The image, together with its blurred version, was called "primal sketch" by Lindeberg (1992, 1993, 1994). Since multi-scale information can be ordered, one obtains a hierarchy representing the subsequent simplification of the image with increasing scale. In one dimensional images critical points can only vanish. Investigation of these locations has been done by several authors (Berkner, 1999; Clark, 1988; Johansen, 1994; Johansen et al., 1986, 2000; Wada and Sato, 1990; Zhao, 1985; Zhao and Iijima, 1985a, 1985b). Higher dimensional images are more complicated as we will discuss below.

\subsection{Related Work}

An essentially unsolved problem in the investigation of deep structure is how to establish meaningful links across scales. A well-defined and user-independent method is obtained by linking points that satisfy some topological constraint. Thus maxima are linked to maxima, etc. This yields so-called critical curves. This approach has been used in 2-D images by various authors (Griffin et al., 1992; Lifshitz and Pizer, 1990; Simmons et al., 1998). They linked extrema, but noticed that sometimes new extrema emerged, disrupting a good linking.
This creation of new extrema in scale space has been studied in detail by Damon (1995, 1997a, 1997b), proving that these creations are generic in images of dimension larger than one. That means that they are not some kind of artifact, introduced by noise or numerical errors, but that they are to be expected in any typical case. This was somewhat counterintuitive, since blurring seemed to imply that structure could only disappear, thus suggesting that only annihilations could occur. Damon, however, showed that both annihilations and creations are generic catastrophes. Furthermore, in (1995) he gave a complete list of local perturbations of these generic events. Johansen (1997) derived the same results by investigating the behaviour of critical curves in scale space. Griffin (1997) investigated critical point events in an affine scale space.

Whereas Damon's results were stated theoretically, application of these results were reported in e.g. (Griffin and Colchester, 1995; Henkel, 1995; Kuijper and Florack, 1999; Lifshitz and Pizer, 1990; Lindeberg, 1992).

Kalitzin (1997) gave artificial examples to show that the methodology of winding numbers (used for detecting critical and degenerated points) was able to detect more complicated catastrophes. The generic catastrophes for certain specified features in an image have also been studied (Eberly et al., 1994; Keller, 1999; Olsen and Nielsen, 1997; Olsen, 1997, 2000; Rieger, 1992, 1995, 1997).

The main outcome of the investigation of the generic results is that in order to be able to use the topological approach, one necessarily needs to take into account both the annihilation and creation events. This has been done in previous work by the authors Kuijper and Florack (2001a, 2001b, 2002), and Kuijper et al. (2003), giving rise to a hierarchical description of the image.

\subsection{Aim}

In images the location of critical points can be found up to the numerical precision of the image. The same holds for the location of catastrophe points in scale space. So although the appearance of catastrophe events can be uniquely separated in annihilations or creations of pairs of critical points, due to e.g. numerical limitations, (almost) symmetries in the image, or coarse sampling also indistinguishable compounds of these annihilation and creation events can be found in practise. In this way 
a couple of nearby generic events may well look like a single, non-generic one.

In this paper we describe these so-called non-generic catastrophes in scale space. The investigation is based on the description of the evolution of critical points in scale space, called (scale space) critical curves, in the neighbourhood of the catastrophe point(s). The compounds of generic events can be modelled using descriptions of "Catastrophe Theory". Obviously, the models obey the property that assuming infinite precision, in non-generic compounds the generic events can be distinguished.

Furthermore, we investigate the appearance of creations as described by these models in more detail and explain why they are, albeit generic, rarely found, probably the reason for current applications to simply ignore them. The results of this investigation give insight in the behaviour of the critical curves and provide sufficient information to establish a well-defined linking of critical points across scales.

The paper is organised as follows: In Section 2 a review of relevant theory on Gaussian scale space and catastrophe theory is given, as well as the way to combine them. Our non-generic catastrophe models in scale space in generic coordinates and their impact on the critical curves are presented in Section 3 . We give some applications in Section 4 and end with a summarising discussion in Section 5.

\section{Theoretical Background}

We define a Gaussian scale space in Section 2.1. The topological change as the scale varies, is called Catastrophe Theory. A summary of relevant theory is presented in Section 2.2. The embedding of Catastrophe Theory in scale space and the generic events are discussed in Section 2.3.

\subsection{Gaussian Scale Space}

Definition 1. $\quad L(\mathbf{x})$ denotes an arbitrary $n$-dimensional image. We will refer to this image as the initial image.

Definition 2. $\quad L(\mathbf{x} ; t)$ denotes the $(n+1)$-dimensional Gaussian scale space image of $L(\mathbf{x})$.

The Gaussian scale space image is obtained by convolution of an initial image with a normalised Gaussian kernel $G(\mathbf{x} ; t)$ of zero mean and standard deviation $\sqrt{2 t}$ :

$L(\mathbf{x} ; t)=(G * L)(\mathbf{x} ; t) \stackrel{\text { def }}{=} \int \frac{1}{\sqrt{4 \pi t}^{n}} e^{-\frac{|\mathbf{x}-\mathbf{y}|^{2}}{4 t}} L(\mathbf{y}) d \mathbf{y}$.

Differentiation is now well-defined. By using multiindex notation for $\alpha=\left(\alpha_{1}, \ldots, \alpha_{n+1}\right), \alpha_{k} \in \mathbb{N}$, take $|\alpha|=\sum_{k=1}^{n+1} \alpha_{k}, \partial^{\alpha}=\partial_{1}^{\alpha_{1}} \partial_{2}^{\alpha_{2}} \ldots \partial_{n+1}^{\alpha_{n+1}}, \partial_{i}=\frac{\partial}{\partial x_{i}}$, and $\partial_{t}=\frac{\partial}{\partial t}$. Then derivatives of the image up to arbitrary order $|\alpha|$ at any scale $t$ are given by

$$
\partial^{\alpha} L=\partial^{\alpha}(G * L)=\left(\partial^{\alpha} G\right) * L .
$$

That is, an arbitrary derivative of the image is obtained by the convolution of the initial image with the corresponding derivative of a Gaussian. Consequently, $L(\mathbf{x} ; t)$ satisfies the diffusion equation:

$$
\partial_{t} L(\mathbf{x} ; t)=\Delta L(\mathbf{x} ; t) .
$$

Here $\Delta L(\mathbf{x} ; t)$ denotes the Laplacean.

Definition 3. Spatial critical points, i.e. saddles and extrema (maxima or minima), at a certain scale $t_{0}$ are defined as the points at fixed scale $t_{0}$ where the spatial gradient vanishes: $\nabla L\left(\mathbf{x} ; t_{0}\right)=0$.

The type of a spatial critical point is given by the eigenvalues of the Hessian $H_{L}$, the matrix with the second order spatial derivatives, evaluated at its location.

Definition 4. The Hessian matrix of $L$ at a certain scale $t_{0}$ is defined by $H_{L} \stackrel{\text { def }}{=} \nabla \nabla^{T} L\left(\mathbf{x} ; t_{0}\right)$, where each element of $H_{L}$ is given by

$$
H_{i, j}=\frac{\partial^{2}}{\partial x_{i} \partial x_{j}} L(\mathbf{x} ; t) .
$$

The trace of the Hessian equals the Laplacean. For maxima (minima) all eigenvalues of the Hessian are negative (positive). At a spatial saddle point $H_{L}$ has both negative and positive eigenvalues.

Since $L(\mathbf{x} ; t)$ is a smooth function in $(\mathbf{x} ; t)$-space, spatial critical points are part of a one dimensional manifold in scale space by virtue of the implicit function theorem.

Definition 5. A critical curve is a one-dimensional manifold in scale space on which $\nabla L(\mathbf{x} ; t)=0$. 
Consequently, the intersection of all critical curves in scale space with a plane of certain fixed scale $t_{0}$ yields the spatial critical points of the image at that scale.

\subsection{Catastrophe Theory}

The spatial critical points of a function with non-zero eigenvalues of the Hessian are called Morse critical points. The Morse Lemma, see e.g., Gilmore (1981), states that at these points the qualitative properties of the function are determined by the quadratic part of the Taylor expansion of this function. This part can be reduced to the Morse canonical form by a slick choice of coordinates.

If at a spatial critical point the Hessian degenerates, so that at least one of the eigenvalues is zero, the type of the spatial critical point cannot be determined by the quadratic part of the Taylor expansion.

Definition 6. The catastrophe points of $L\left(\mathbf{x} ; t_{0}\right)$ are defined as the points where both the spatial gradient and the determinant of the Hessian vanish: $\nabla L\left(\mathbf{x} ; t_{0}\right)=0$ and $\operatorname{det} H_{L}\left(\mathbf{x} ; t_{0}\right)=0$.

The term catastrophe was introduced by Thom (1972, 1975). It denotes a (sudden) qualitative change in an object as the parameters on which this object depends change smoothly. This behaviour was already known by the terms perestroika, bifurcation and metamorphosis. The name catastrophe theory was suggested by Zeeman (1977) to unify singularity theory, bifurcation theory and their applications and gained wide popularity. A thorough mathematical treatment on singularity theory can be found in the work of Arnol'd (1983, 1984, 1992, 1993a, 1993b, 1994). More pragmatic introductions and applications are widely published (e.g., Bruce and Giblin, 1984; Fomenko and Kunii, 1997; Gilmore, 1981; Lu, 1976; Poston and Stewart, 1981; Woodcock and Davis, 1978; Zeeman, 1977).

The catastrophe points are also called non-Morse critical points, since a higher order Taylor expansion is essentially needed to describe the qualitative properties. Although the dimension of the variables is arbitrary, the Thom Splitting Lemma, see e.g. Gilmore (1981), states that one can split up the function in a Morse and a non-Morse part. The latter consists of variables representing the $k$ "bad" eigenvalues of the Hessian that become zero. The Morse part contains the $n-k$ remaining variables. Consequently, the Hessian contains a $(n-k) \times(n-k)$ sub-matrix representing a Morse function. It therefore suffices to study the part of $k$ variables. The canonical form of the function at the non-Morse critical point thus contains two parts: a Morse canonical form of $n-k$ variables, in terms of the quadratic part of the Taylor series, and a non-Morse part. The latter can be put into canonical form called the catastrophe germ, which is a polynomial of degree 3 or higher.

Since the Morse part does not change qualitatively under small perturbations, it is not necessary to further investigate this part. The non-Morse part, however, does change. Generally the non-Morse critical point will split into a non-Morse critical point, described by a polynomial of lower degree, and Morse critical points, or even exclusively into Morse critical points. This event is called a morsification. So the non-Morse part contains the catastrophe germ and a perturbation that controls the morsifications.

Then the general form of a Taylor expansion $f(\mathbf{x})$ at a non-Morse critical point of an $n$ dimensional function can be written as (Thom's Theorem):

$$
\begin{aligned}
f(\mathbf{x} ; \lambda)= & C G\left(x_{1}, \ldots, x_{k}\right) \\
& +P T\left(x_{1}, \ldots, x_{k} ; \lambda_{1}, \ldots, \lambda_{l}\right)+\sum_{i=k+1}^{n} \epsilon_{i} x_{i}^{2},
\end{aligned}
$$

where $C G\left(x_{1}, \ldots, x_{k}\right)$ denotes the catastrophe germ, $P T\left(x_{1}, \ldots, x_{k} ; \lambda_{1}, \ldots, \lambda_{l}\right)$ the perturbation germ with an $l$-dimensional space of parameters, and in the Morse part $\epsilon_{i}= \pm 1$.

The so-called simple real singularities in $2 \mathrm{D}$, so $\mathbf{x}=$ $(x, y)$, have catastrophe germs given by the two infinite series

- $A_{k}^{ \pm} \stackrel{\text { def }}{=} \pm x^{k+1}, k \geq 1$. The germs $A_{k}^{+}$and $A_{k}^{-}$are equivalent for $k=1$ and $k>2, k$ even.

- $D_{k}^{ \pm} \stackrel{\text { def }}{=} x^{2} y \pm y^{k-1}, k \geq 4$, which we will rewrite to $x^{k-1} \pm x y^{2}$ for notational convenience.

Furthermore the three so-called "exceptional singularities" occur:

$-E_{6} \stackrel{\text { def }}{=} x^{3} \pm y^{4}$

$-E_{7} \stackrel{\text { def }}{=} x^{3}+x y^{3}$, and

$-E_{8} \stackrel{\text { def }}{=} x^{3}+y^{5}$.

\subsection{Catastrophes and Scale Space}

In Definition 6, the number of equations defining the catastrophe point equals $n+1$ and therefore it is overdetermined with respect to the $n$ spatial variables. 
Consequently, catastrophe points are generically not found in typical images. In scale space, however, the number of variables equals $n+1$ and catastrophes occur as isolated points.

Although the list of catastrophes starts very simple, it is not trivial to apply it directly to scale space by assuming that scale is just one of the perturbation parameters. As Damon (1995, 1997b) points out:

"There are significant problems in trying to directly apply Morse theory to solutions of to the heat equation. First, it is not clear that generic solutions to the heat equation must be generic in the Morse sense. Second, standard models for Morse critical points and their annihilation and creation do not satisfy the heat equation. How must these models be modified? Third, there is the question of what constitutes generic behaviour. This depends on what notion of local equivalence one uses between solutions to the heat equation."

For example, in one-dimensional images the $A_{2}$ catastrophe reduces to $x^{3}+\lambda x$. It describes the change from a situation with two critical points (a maximum and a minimum) for $\lambda<0$ to a situation without critical points for $\lambda>0$. This event can occur in two ways. The extrema are annihilated for increasing $\lambda$, but the opposite-creation of two extrema for decreasing $\lambda$ is also possible.

In scale space, however, there is an extra constraint: the germ has to satisfy the diffusion equation. Thus the catastrophe germ $x^{3}$ implies an extra term $6 x t$. On the other hand, the perturbation term is given by $\lambda_{1} x$, so by taking $\lambda_{1}=6 t$ scale plays the role of the perturbing parameter. This gives a directionality to the perturbation parameter, in the sense that the only remaining possibility for this $A_{2}$-catastrophe in one-dimensional images is an annihilation. So the Fold catastrophe is adjusted such that it satisfies the heat equation, but this adjustment only allows annihilations. However, it does not imply that only annihilations are generic in scale space. In higher dimensional images also the opposite-i.e. a $A_{2}$ catastrophe describing the creation of a pair of critical points-is possible. Then the perturbation $\lambda=-6 t$ with increasing $t$ requires an additional term of the form $-6 x y^{2}$ in order to satisfy the diffusion equation as we will see.

The transfer of the catastrophe germs to scale space has been made by many authors (Damon, 1995, 1997a, 1997b; Florack and Kuijper, 2000; Johansen, 1997a; Johansen et al., 1986, 2000; Kalitzin, 1997; Kalitzin et al., 1998; Kuijper and Florack, 1999, 2001a, 2001b, 2002; Kuijper et al., 2003, Lindeberg, 1992, 1994), among whom Damon's account is probably the most rigorous. He showed that the only generic morsifications in scale space are the aforementioned $A_{2}$ catastrophes describing annihilations and creations of pairs of critical points. These two points have opposite sign of the determinant of the Hessian before annihilation and after creation. All other events are compounds of such events. It is however possible that one may not be able to distinguish these generic events, e.g. due to numerical limitations, coarse sampling, or (almost) symmetries in the image. For instance, one may find at some scale three nearby critical points, e.g. two extrema and a saddle, and at the subsequent scale only one extremum. Obviously, one pair of critical points is annihilated, but one may not be able to identify the annihilating extremum at the former scale. This is illustrated in Fig. 1. On the left the critical paths are shown, together with a grey area representing the uncertainty in determining the catastrophe location. On the right the non-generic model of this event is displayed.

Definition 7. The scale space catastrophe germs are defined by

$$
\begin{aligned}
& f^{\mathrm{A}}(\mathbf{x} ; t) \stackrel{\text { def }}{=} x_{1}^{3}+6 x_{1} t+Q(\tilde{\mathbf{x}} ; t), \\
& f^{\mathrm{C}}(\mathbf{x} ; t) \stackrel{\text { def }}{=} x_{1}^{3}-6 x_{1} t-6 x_{1} x_{2}^{2}+Q(\tilde{\mathbf{x}} ; t) .
\end{aligned}
$$

The quadratic term $Q(\tilde{\mathbf{x}} ; t)$ is defined

$$
Q(\tilde{\mathbf{x}} ; t) \stackrel{\text { def }}{=} \sum_{i=2}^{n} \epsilon_{i}\left(x_{i}^{2}+2 t\right)
$$

where $\sum_{i=2}^{n} \epsilon_{i} \neq 0$ and $\epsilon_{i} \neq 0 \forall i$.

Note that the scale space catastrophe germs $f^{\mathrm{A}}$ and $f^{\mathrm{C}}$, and the quadratic term $Q$ satisfy the diffusion equation. The germs $f^{\mathrm{A}}$ and $f^{\mathrm{C}}$ correspond to the
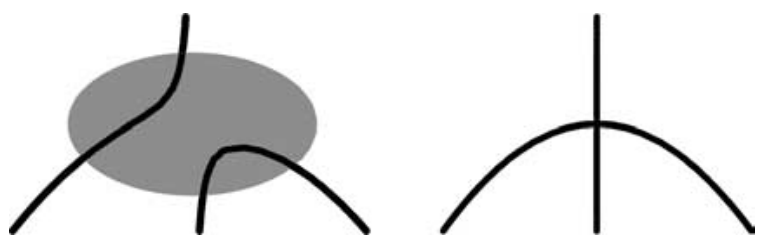

Figure 1. Left: Annihilation of two critical points in the neighbourhood of a third critical point. The grey area represents the uncertainty in determining the catastrophe. Right: Non-generic representation and model of this event. 
two qualitatively different $A_{2}$ catastrophes at the origin, an annihilation and a creation respectively. From Definition 7 it is obvious that annihilations occur in any dimension, but creations require at least 2 dimensions. Consequently, in 1D signals only annihilations occur. Furthermore, for images of arbitrary dimension and fewer than three vanishing eigenvalues of the Hessian at a degenerated point, it suffices to investigate the 2D case due to the Splitting Lemma.

2.3.1. The Annihilation Germ. Spatial critical points at any scale $t$ for $f^{\mathrm{A}}$ follow directly from $\nabla f^{\mathrm{A}}(\mathbf{x} ; t)=0$ :

$$
\left\{\begin{aligned}
3 x_{1}^{2} & =-6 t \\
2 \epsilon_{i} x_{i} & =0, \quad i \geq 2
\end{aligned}\right.
$$

Then the critical curve is parametrised by $( \pm \sqrt{-2 t}, 0, \ldots, 0 ; t), t \leq 0$. At the origin a catastrophe takes place. The determinant of the Hessian is given by $\operatorname{det} H_{f^{\mathrm{A}}}=c x_{1}$, with the constant $c=3 \cdot 2^{n}$ $\prod_{i=2}^{n} \epsilon_{i}$. So two critical points with opposite sign approach the origin as $t$ increases to zero and meet transversally to the Hessian zero-crossing. Note that $\operatorname{tr} H_{f^{\mathrm{A}}}=6 x_{1}+\sum_{i=2}^{n} 2 \epsilon_{i}$, which is generically non-zero at catastrophe points. This explains the constraints on the $\epsilon_{i}$ in Definition 7. It implies that generically catastrophe points are not scale space critical points, i.e. points with vanishing gradient and vanishing scale derivative.

2.3.2. The Creation Germ. The creation germ is a bit more complicated. Spatial critical points at any scale $t$ for $f^{\mathrm{C}}$ follow from $\nabla f^{\mathrm{C}}(\mathbf{x} ; t)=0$ :

$$
\left\{\begin{aligned}
3 x_{1}^{2}-6 x_{2}^{2} & =6 t \\
2 x_{2}\left(\epsilon_{2}-6 x_{1}\right) & =0 \\
2 \epsilon_{i} x_{i} & =0, \quad i \geq 3
\end{aligned}\right.
$$

Since we look in the neighbourhood of the origin, we take $x_{2}=0$. Then the critical curve is parametrised by $( \pm \sqrt{2 t}, 0, \ldots, 0 ; t), t \geq 0$. At the origin a catastrophe takes place. The determinant of the Hessian is given by $\operatorname{det} H_{f} \mathrm{c}=c x_{1}\left(\epsilon_{2}-6 x_{1}\right)-12 c x_{2}^{2}$, with the constant $c=3 \cdot 2^{n} \prod_{i=3}^{n} \epsilon_{i}$, so two critical points with opposite sign leave the origin as $t$ increases from zero, again transversally to the Hessian zero-crossing. Note that this catastrophe is a $A_{2}$ catastrophe, since it describes the creation of two critical points, although there is a striking resemblance to the description of the $D_{4}^{-}$catastrophe. Furthermore, the description of the catastrophe is essentially local: If $t$ is taken too large, the (non- generic) degeneration of the Hessian at $x_{1}=\frac{1}{6} \epsilon_{2}$ has to be taken into account. We will elaborate on these items in Section 3.

\section{Scale Space Catastrophe Models}

In this section we describe how we will use the following catastrophes (with nicknames and perturbation germs) to model events in $(2+1)$-dimensional scale space. The catastrophes describe in canonical coordinates how critical curves pass the origin yielding compounds of annihilations and / or creations of pairs of critical points.

- $A_{2}$ Fold catastrophe: $x^{3}+\lambda_{1} x \pm y^{2}$.

- $A_{3}$ Cusp catastrophe: $\pm x^{4}+\lambda_{1} x+\lambda_{2} x^{2} \pm y^{2}$.

- $D_{4}^{+}$Hyperbolic Umbilic catastrophe, $x^{3}+x y^{2}+$ $\lambda_{1} x+\lambda_{2} y+\lambda_{3} y^{2}$.

- $D_{4}^{-}$Elliptic Umbilic catastrophe: $x^{3}-x y^{2}+\lambda_{1} x+$ $\lambda_{2} y+\lambda_{3} y^{2}$.

- $D_{5}^{ \pm}$Parabolic Umbilic catastrophe: $x^{4} \pm x y^{2}+\lambda_{1} x+$ $\lambda_{2} y+\lambda_{3} y^{2}+\lambda_{4} x^{2}$.

- $D_{6}^{+}$Second Hyperbolic Umbilic catastrophe: $x^{5}+$ $x y^{2}+\lambda_{1} x+\lambda_{2} y+\lambda_{3} x^{2}+\lambda_{4} y^{2}+\lambda_{5} x^{3}$.

- $D_{6}^{-}$Second Elliptic Umbilic catastrophe: $x^{5}-x y^{2}+$ $\lambda_{1} x+\lambda_{2} y+\lambda_{3} x^{2}+\lambda_{4} y^{2}+\lambda_{5} x^{3}$.

The \pm signs at the $A_{3}$ and $D_{5}$ denote 'dual' possibilities with similar geometry.

Again we emphasise that this list not a complete list as can be found in Damon (1995), containing all the relevant mathematical details. However, the germs mentioned above are related to the perturbations of the generic annihilation and creation given in Damon (1995). We will see that although most of these catastrophes are non-generic, they may still be relevant for modelling compounds of generic events that one is not capable of, or willing to, segregate as such. Recall, for example (Fig. 1).

The germs in this list are adjusted such that they satisfy the heat equation. Furthermore, by choosing the perturbation terms non-zero and adjusting them in the same way, descriptions of critical curves in scale space are obtained. These critical curves only contain the generic Fold annihilation(s) and/or creation(s).

\section{1. $A_{2}$ Fold Catastrophe}

The Fold catastrophe in scale space is given by

$$
F(x, y ; t)=x^{3}+6 x t+\delta\left(y^{2}+2 t\right),
$$


where $\delta= \pm 1$. Critical curves and the catastrophe point follow from

$$
\left\{\begin{aligned}
\partial_{x} F & =3 x^{2}+6 t \\
\partial_{y} F & =2 \delta y \\
\operatorname{det}\left(H_{F}\right) & =12 \delta x .
\end{aligned}\right.
$$

One can verify that at the origin a saddle and an extremum (a minimum if $\delta=1$, a maximum if $\delta=-1$ ) moving in the $y=0$ plane meet and annihilate while increasing the scale parameter $t$.

\section{2. $A_{3}$ Cusp Catastrophe}

The Cusp catastrophe germ is given by $x^{4}$. Its scale space addition is $12 x^{2} t+12 t^{2}$. The perturbation term contains two terms: $\lambda_{1} x+\lambda_{2} x^{2}$. Obviously, scale takes the role of $\lambda_{2}$. Taking the dual Cusp gives the same geometry by changing the sign of $\lambda_{1}$, or by setting $x=-x$. The scale space Cusp catastrophe germ with perturbation is thus defined by

$C(x, y ; t)=x^{4}+12 x^{2} t+12 t^{2}+\lambda_{1} x+\delta\left(y^{2}+2 t\right)$,

with $\delta= \pm 1$. Again, the critical curves and the catastrophe point follow from

$$
\left\{\begin{aligned}
\partial_{x} C & =4 x^{3}+24 x t+\lambda_{1} \\
\partial_{y} C & =2 \delta y \\
\operatorname{det}\left(H_{C}\right) & =24 \delta\left(x^{2}+2 t\right) .
\end{aligned}\right.
$$

Morsification by the perturbation $\lambda_{1} \neq 0$ yields one Fold catastrophe and one regular critical curve, see Fig. 2(a) and (c). The differences in behaviour depending on the sign of $\delta$ is studied in detail in Kuijper and Florack (2001b). It suffices here to note that if $\lambda_{1}=0$, at the origin three critical points transform to one critical point while increasing scale, see Fig. 2(b).

One can verify that the $A_{k}, k>3$ catastrophes describe the (non-generic) simultaneous annihilations of critical points in one dimension under the influence of blurring, albeit in more complicated appearances.

\section{3. $D_{4}^{ \pm}$Umbilic Catastrophes}

The $D_{4}^{ \pm}$Umbilic catastrophe germs are given by $x^{3}+\delta x y^{2}$, where $\delta= \pm 1$. The scale space addition is $(6+2 \delta) x t$, yielding $x^{3}+x y^{2}+8 x t$ for the Hyperbolic Umbilic catastrophe, and $x^{3}-x y^{2}+4 x t$ for the Elliptic Umbilic catastrophe. The perturbation contains three terms: $\lambda_{1} x+\lambda_{2} y+\lambda_{3} y^{2}$. Obviously, scale takes the role of $\lambda_{1}$ and the $\lambda_{3}$ term becomes $l a_{3}\left(y^{2}+2 t\right)$. So the scale space Umbilic catastrophe germs with perturbation are given by

$$
\begin{aligned}
U(x, y ; t)= & x^{3}+6 x t+\delta\left(x y^{2}+2 x t\right) \\
& +\lambda_{3}\left(y^{2}+2 t\right)+\lambda_{2} y .
\end{aligned}
$$

3.3.1. $D_{4}^{ \pm}$Hyperbolic Umbilic Catastrophe. The scale space $D_{4}^{+}$Hyperbolic Umbilic catastrophe germ with perturbation follows from Eq. (5) by taking $\delta=1$ and is thus defined by

$H U(x, y ; t)=x^{3}+x y^{2}+8 x t+\lambda_{3}\left(y^{2}+2 t\right)+\lambda_{2} y$.

The critical curves and catastrophe points follow from

$$
\left\{\begin{aligned}
\partial_{x} H U & =3 x^{2}+8 t+y^{2} \\
\partial_{y} H U & =2 x y+2 \lambda_{3} y+\lambda_{2} \\
\operatorname{det}\left(H_{H U}\right) & =12 x\left(x+\lambda_{3}\right)-4 y^{2} .
\end{aligned}\right.
$$

In the unperturbed situation four critical points exist for each $t<0$ on the $x$ - and $y$-axes. At $t=0$ the four critical curves annihilate simultaneously at the origin, see

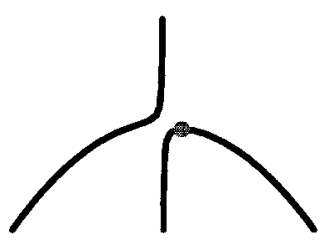

a) $\lambda_{1}=1$.

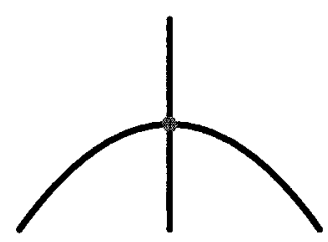

b) $\lambda_{1}=0$.

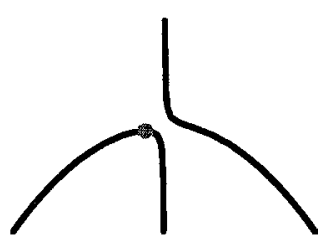

c) $\lambda_{1}=-1$.

Figure 2. Critical paths of the Cusp catastrophe in $(x ; t)$-space $(y=0)$. The catastrophe is located at the dot. Note that if the perturbation is very small, these three distinct cases may very well be confused. 


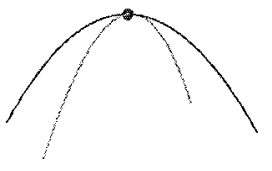

a) Unperturbed.

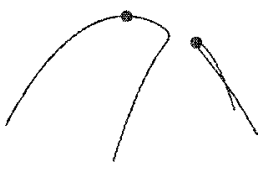

b) Perturbed.
Figure 3. Critical paths of the $D_{4}^{+}$-catastrophe in $(x, y ; t)$-space. Again, if the perturbation is small we may not be able to tell which configuration is the actual one.

Fig. 3(a). Taking perturbation into account, the curves are separated into two critical curves each containing a Fold catastrophe, see Fig. 3(b).

3.3.2. $\mathrm{D}_{4}^{-}$Elliptic Umbilic Catastrophes. The scale space elliptic Umbilic catastrophe germ with perturbation follows from Eq. (5) by taking $\delta=-1$ and is given by

$$
E U(x, y ; t)=x^{3}-x y^{2}+4 x t+\lambda_{3}\left(y^{2}+2 t\right)+\lambda_{2} y .
$$

Again, the critical curves and the catastrophe points follow from

$$
\left\{\begin{aligned}
\partial_{x} E U & =6 x^{2}+4 t-y^{2} \\
\partial_{y} E U & =-2 x y+2 \lambda_{3} y+\lambda_{2} \\
\operatorname{det}\left(H_{E U}\right) & =12 x\left(2 \lambda_{3}-2 x\right)-4 y^{2} .
\end{aligned}\right.
$$

The unperturbed equation gives two critical points for all $t \neq 0$. At the origin a so-called scatter event occurs: the critical curve changes from $y$-axis to $x$-axis with increasing $t$, see Fig. 4. Just as in the hyperbolic case, in fact two Fold catastrophes take place; in this case both an annihilation and a creation. The morsification is shown in Fig. 4(b). The critical curve on the right does not contain catastrophe points. The critical curve on the left, however, contains two Fold catastrophe points: a creation and an annihilation. Both are studied in detail in Kuijper and Florack (2001b) in relation to the hierarchical structure of the scale space

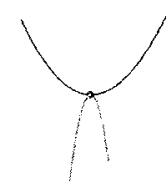

a) Unperturbed.

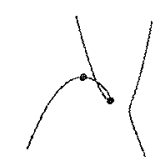

b) Small perturbation.

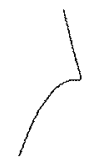

c) Large perturbation.
Figure 4. Critical paths of the $D_{4}^{-}$-catastrophe in $(x, y ; t)$-space. image as mentioned in the introduction. A brief sketch was given in Section 2, where we could set $\lambda_{2}=0$, since only the creation at the origin was investigated.

So while increasing scale one will find two critical points, suddenly two extra critical points appear, of which one annihilates with one of the already existing ones. Finally, one ends up with again two critical points. Clearly, if the samples in scale are taken too large, one could completely miss the subsequent catastrophes, see e.g. Fig. 4(c). ${ }^{1}$ The properties of the creations will be discussed in the next section.

3.3.3. Creations. As we showed, a creation event can occur in case of a morsified scale space elliptic Umbilic catastrophe. $^{2}$ In most applications, however, creations are rarely found, giving rise to the (false) opinion that creations are caused by numerical errors and should be disregarded. The reason for their rare appearance lies in the specific requirements for the parameters in the (morsified) Umbilic catastrophe germ. Its general formulation is given by

$$
\begin{aligned}
U(x, y ; t)= & \frac{1}{6} L_{x x x} x^{3}+\frac{1}{2} L_{x y y} x y^{2}+L_{x t} x t \\
& +\frac{1}{2} L_{y y}\left(y^{2}+2 t\right)+L_{y} y
\end{aligned}
$$

In general, the spatial coefficients do not equal the derivatives evaluated in the coordinate system of the image. They follow from the alignment of the catastrophe in the plane defined by $y=0$ and can have arbitrary value. ${ }^{3}$ Furthermore, recall that the diffusion equation implies $L_{x t} \stackrel{\text { def }}{=} L_{x x x}+L_{x y y}$. Then the scale space evolution of the critical curves follow from

$$
\left\{\begin{aligned}
\partial_{x} U & =\frac{1}{2} L_{x x x} x^{2}+L_{x t} t+\frac{1}{2} L_{x y y} y^{2} \\
\partial_{y} U & =L_{x y y} x y+L_{y y} y+L_{y} \\
\operatorname{det}\left(H_{U}\right) & =L_{x x x} x\left(L_{x y y} x+L_{y y}\right)-L_{x y y}^{2} y^{2} .
\end{aligned}\right.
$$

Firstly we consider the case $L_{y}=0$. Then Eq. (8) describes a Fold catastrophe (either annihilation or creation) at the origin, where the critical curve is positioned in the $(x, t)$-plane. A creation necessarily requires the constraint $L_{x x x} L_{x t}<0$ at the catastrophe point. This constraint is sufficient.

Theorem 1. At a catastrophe point in two spatial dimensions, if the third order derivatives of the general local form as given by Eq. (8) with $L_{y}=0$ are uncorrelated, the number of creations has an a priori 
likelihood of $1 / 4$ relative to the total number of catastrophes. In $n$ dimensions it is $\frac{1}{\pi} \arccos \frac{1}{\sqrt{n}}$.

Proof: The requirement $L_{x x x} L_{x t}<0$ can be rewritten to $L_{x x x}\left(L_{x x x}+L_{x y y}\right)<0$. In the $\left(L_{x x x}, L_{x y y}\right)$ space this constraint is satisfied by all point sets in the two areas bounded by the lines from $L_{x x x}=0$ to $L_{x x x}+L_{x y y}=0$ over an angle $\phi$. This is exactly the angle between the normal vectors to the lines, viz. $(1,0)$ and $(1,1)$, locating a quarter of the plane.

For $n \mathrm{D}$ this extends to the fraction of $\left(L_{x x x}, L_{x y_{i} y_{i}}\right)$ space, with $\operatorname{dim}(\mathbf{y})=n-1$, where $L_{x x x}\left(L_{x x x}+L_{x y_{1} y_{1}}+\right.$ $\left.\cdots+L_{x y_{n-1} y_{n-1}}\right)<0$. This represents two intersecting hyperplanes with normal vectors $(1,0, \ldots, 0)$ and $(1,1, \ldots, 1)$ over an angle $\phi$, given by

$$
\cos \phi=\frac{(1,0, \ldots, 0) \cdot(1,1, \ldots, 1)}{|(1,0, \ldots, 0)| \cdot|(1,1, \ldots, 1)|}=\frac{1}{\sqrt{n}}
$$

Then the fraction of the space follows by taking twice this angle and dividing by the complete angle of $2 \pi$, i.e. $\frac{1}{\pi} \arccos \frac{1}{\sqrt{n}}$.

Note that if $n=1$, the fraction of the space where creations can occur is (indeed) zero, and for $n=2$ it is a quarter. The also interesting case $n=3$ yields a fraction that is slightly more than a quarter, whereas for $n \rightarrow \infty$ the fraction converges to a half, see Fig. 5(a). That is: the higher the dimensions, the easier critical points can be created.

The reason that in practice in two dimensional images the number of creations observed is (much) smaller than a quarter, is caused by the role of the perturbation parameters. It is possible to give a tight bound to the perturbation of Eq. (8) in terms of $L_{y}$ :
Theorem 2. A creation and subsequent annihilation event occur in Eq. (8) if and only if

$$
\left|L_{y}\right| \leq \frac{3}{16} L_{y y}^{2} \sqrt{\frac{-3 L_{x x x}}{L_{x y y}^{3}}}
$$

Proof: The catastrophes satisfy $\partial_{x} L=\partial_{y} L=\operatorname{det}$ $H=0$. Since the system

$$
\begin{aligned}
\partial_{y} L & =L_{y}+y\left(L_{y y}+L_{x y y} x\right)=0 \\
\operatorname{det} H_{U} & =L_{x x x} x\left(L_{y y}+L_{x y y} x\right)-L_{x y y}^{2} y^{2}=0
\end{aligned}
$$

contains only spatial coordinates, the intersections of these implicitly given curves define the spatial coordinates of the catastrophes. The catastrophe points form the local extrema of the critical curve in $(x, y ; t)$-space, i.e. at these points the tangent vector has no scale component. If the curves given by Eq. (10) touch, there is only a point of inflection in the critical curve, i.e. the critical curve in $(x, y ; t)$-space has a (Fold) catastrophe point. At this point of inflection, the spatial tangent vectors of the curves defined by Eq. (10) are equal.

Solving Eq. (10) with respect to $y$ results in

$$
y=-\frac{L_{y}}{L_{y y}+L_{x y y} x}= \pm \frac{1}{L_{x y y}} \sqrt{L_{x x x} x\left(L_{y y}+L_{x y y} x\right)} .
$$

The equality of the tangent vectors at the point of inflection $x_{i}, y_{i}$ yields

$$
\begin{aligned}
& \left.\frac{\partial}{\partial x}\left(-\frac{L_{y}}{L_{y y}+L_{x y y} x}\right)\right|_{x_{i}, y_{i}} \\
& \quad=\left.\frac{\partial}{\partial x}\left( \pm \frac{1}{L_{x y y}} \sqrt{L_{x x x} x\left(L_{y y}+L_{x y y} x\right)}\right)\right|_{x_{i}, y_{i}}
\end{aligned}
$$

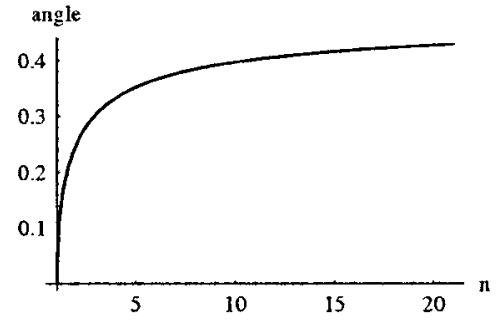

a) Space fraction.

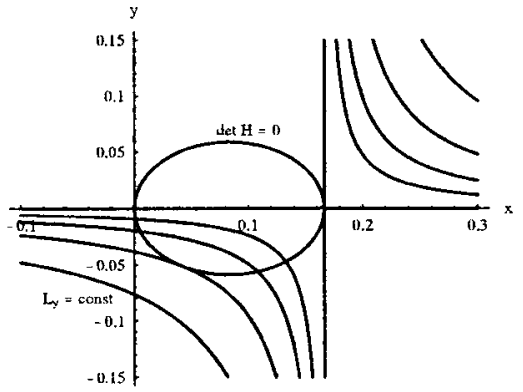

b) Dependency on $L_{y}$.

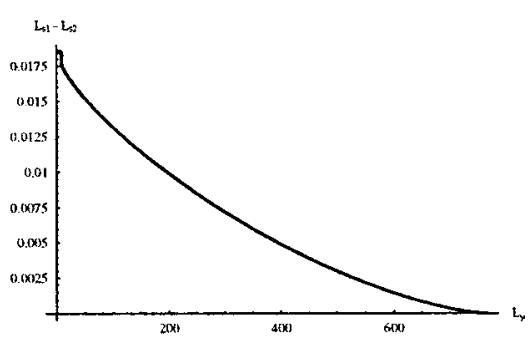

c) Intensity difference.

Figure 5. (a) The fraction of the space of the third order derivatives in which creations can occur as a function of the dimension according to Theorem 1. (b) Intersections of the curves $\operatorname{det}(H)=0$ and $\partial_{y} L=0$ with different values for $L_{y}$. For the value given by Theorem 2 the curves touch. (c) Difference in intensity between the creation and the annihilation event for $L_{y}$ increasing from 0 to its critical value. 
Solving both equalities results in

$$
\begin{aligned}
&\left(x_{i}, y_{i}, L_{y}\right)=\left(-\frac{L_{y y}}{4 L_{x y y}}, \pm \sqrt{\frac{-3 L_{x x x} L_{y y}^{2}}{16 L_{x y y}^{3}}},\right. \\
&\left.\mp \frac{3 L_{y y}^{2}}{16 L_{x y y}} \sqrt{\frac{-3 L_{x x x}}{L_{x y y}}}\right),
\end{aligned}
$$

which gives the boundary values for $L_{y}$.

Note that Eq. (9) has only real solutions if $L_{x x x} L_{x y y}<0$, i.e. at the $D_{4}^{-}$(morsified) catastrophe. As a consequence of Theorem 2, creations only occur if the perturbation is small enough. Again, this perturbation occurs in the coordinate system, obtained by the alignment of the catastrophe in the plane defined by $y=0$.

Example 1. Taking $L_{x x x}=6, L_{x y y}=-12, L_{y y}=2$ yielding $U=x^{3}-6 x y^{2}-6 x t+y^{2}+2 t+L_{y}$, we obtain the "generic creation example" as given in Section 2 with perturbation. Then Theorem 2 gives $\left|L_{y}\right| \leq \frac{1}{32} \sqrt{6}$ as a (compared to the other derivative values) relatively small bound for the occurrence of a creationannihilation couple.

In Fig. 5(b) the ellipse $\operatorname{det}\left(H_{U}\right)=0$ is plotted, together with the curves $\partial_{y} U=0$ for $L_{y}=c$. For $c=0$ this results in the two straight lines $y=0$ and $x=\frac{1}{6}$, intersecting at $\left.(x, y)=\left(\frac{1}{6}, 0\right)\right)$. For $c=2^{-i} \sqrt{6}, i=4, \ldots, 7$ the solution gives hyperbolae with the aformentioned lines as asymptotes. If $i>5$, the perturbation is small enough and the intersection of $\partial_{y} U=0$ and $\operatorname{det}\left(H_{U}\right)=0$ contains two points. Thus a creation-annihilation is observed. If $i=5, L_{y}$ has its critical value and the curves touch. For larger values of $c$ the curves do not intersect each other.

Obviously the perturbation $L_{y}$ can be larger if $L_{y y}$ increases. If so, the structure becomes more elongated. It is known by various examples of creations given in literature that elongated structures play an important role. In fact, the quintessential property is scale anisotropy.

Another reason that creations are rarely found is that their lifetime is rather limited: with increasing $t$ the created critical points annihilate. If the scale steps are taken too large, one simply misses the creation - annihilation couple. This may be regarded as a dual expression for the previous explanation. In the chosen coordinate system this can be calculated explicitly.
Theorem 3. The maximum lifetime of a creation given by Eq. (8) is

$$
t_{\text {lifetime }}=\frac{-L_{x x x} L_{y y}^{2}}{2 L_{x y y}^{2}\left(L_{x x x}+L_{x y y}\right)} .
$$

The difference in intensity of the critical point that is created and subsequently annihilated is

$$
\frac{L_{x x x}\left(2 L_{x x x}-L_{x y y}\right) L_{y y}^{3}}{6 L_{x y y}^{3}\left(L_{x x x}+L_{x y y}\right)} .
$$

Proof: Observe that the lifetime is bounded by the distance between the two intersections of $\partial_{y} L=0$ and $\operatorname{det}\left(H_{U}\right)=0$, see Fig. 5(b). As $\left|L_{y}\right|$ increases from zero, the two points move towards each other over the $\operatorname{arc} \operatorname{det}\left(H_{U}\right)=0$ until they reach the value given by Theorem 2 with lifetime equal to zero. The largest arc length is obtained for $L_{y}=0$. Then the spatial coordinates are found by

$$
\partial_{y} L(x, y ; t)=y\left(L_{x y y} x+L_{y y}\right)=0
$$

and

$$
\operatorname{det} H_{U}=L_{x x x} x\left(L_{x y y} x+L_{y y}\right)-L_{x y y}^{2} y^{2}=0,
$$

i.e. $\left(x_{0}, y_{0}\right)=(0,0)$ and $\left(x_{1}, y_{1}\right)=\left(-\frac{L_{y y}}{L_{x y y}}, 0\right)$ The locations in scale space are given by

$$
\partial_{x} L(x, y ; t)=\frac{1}{2} L_{x x x} x^{2}-\frac{1}{2} L_{x y y} y^{2}+L_{x t} t=0 .
$$

Consequently, the first catastrophe takes place at the origin-since also $t_{0}=0$-with $L\left(x_{0}, y_{0}, t_{0}\right)=0$. Noting that $L_{x t}=L_{x x x}+L_{x y y}$ by the diffusion equation, the second one is located at

$$
\left(x_{1}, y_{1} ; t_{1}\right)=\left(-\frac{L_{y y}}{L_{x y y}}, 0 ; \frac{-L_{x x x} L_{y y}^{2}}{2 L_{x y y}^{2}\left(L_{x x x}+L_{x y y}\right)}\right)
$$

with intensity

$$
L\left(x_{1}, y_{1} ; t_{1}\right)=\frac{L_{x x x}\left(2 L_{x x x}-L_{x y y}\right) L_{y y}^{3}}{6 L_{x y y}^{3}\left(L_{x x x}+L_{x y y}\right)} .
$$

The latter is also the maximum difference in intensity $L_{\text {cat }} \stackrel{\text { def }}{=} L\left(x_{1}, y_{1} ; t_{1}\right)-L\left(x_{0}, y_{0}, t_{0}\right)$. 
Example 2. To show the effect of the movement along the $\operatorname{arc} \operatorname{det}\left(H_{U}\right)=0$, see Fig. 5(c). Without loss of generality we took again $L_{x x x}=6, L_{x y y}=$ $-12, L_{y y}=2$. Firstly, the two solutions to $\nabla L=$ $0 \wedge \operatorname{det}\left(H_{U}\right)=0$ were calculated as function of $L_{y}$. Secondly, the difference of the intensity of the solutions was calculated for 766 subsequent values of $L_{y}$, $L_{y} \in\left[0, \ldots, \frac{1}{32} \sqrt{6}\right]$. It is clearly visible that the intensity decreases monotonously with an increase of $L_{y}$. For this example we find that the maximum lifetime is $\frac{1}{72}$, the maximum difference in intensity $\frac{1}{18}$.

From the proof of Theorem 3 it is again apparent that $L_{y y}$ plays an important role in enabling a (long)lasting creation. To observe this in more detail, note that the curve

$$
\operatorname{det} H_{U}=0 \Leftrightarrow L_{x x x} x\left(L_{x y y} x+L_{y y}\right)=L_{x y y}^{2} y^{2}
$$

is an ellipse (see also Fig. 5(b)). Replacing $x$ by $x-$ $\frac{L_{y y}}{2 L_{x y y}}$, it is centred at the origin:

$$
L_{x x x}\left(x-\frac{L_{y y}}{2 L_{x y y}}\right) L_{x y y}\left(x+\frac{L_{y y}}{2 L_{x y y}}\right)=L_{x y y}^{2} y^{2} .
$$

And consequently

$$
L_{x x x} L_{x y y}\left(x^{2}-\frac{L_{y y}^{2}}{4 L_{x y y}^{2}}\right)=L_{x y y}^{2} y^{2} .
$$

Setting $L_{x y y}=\frac{1}{b}$ and $L_{x x x} L_{x y y}=-\frac{1}{a^{2}}$, we find

$$
\operatorname{det} H_{U}=0 \Leftrightarrow x^{2}+\frac{a^{2}}{b^{2}} y^{2}=L_{y y}^{2} \frac{b^{2}}{4} .
$$

Assuming that we have a creation, $a^{2}>0$. The ellipse is enlarged with an increase of $L_{y y}^{2}$. Obviously, at the annihilations of the Hyperbolic Umbilic catastrophe $a^{2}<0$, so $\operatorname{det} H_{U}=0$ then describes a hyperbola.

\section{4. $D_{5}^{ \pm}$Parabolic Umbilic Catastrophes}

In the previous section we saw that the geometry significantly changed by taking either the term $-x y^{2}$, or the term $+x y^{2}$. Let us therefore, ignoring the perturbation terms $\lambda_{1}, \lambda_{2}$, and $\lambda_{3}$, define the scale space Parabolic Umbilic catastrophe germ by

$P U(x, y ; t)=\frac{1}{4 !} x^{4}+\frac{1}{2 !} x^{2} t+\frac{1}{2 !} t^{2}+\delta\left(\frac{1}{2} x y^{2}+x t\right)$

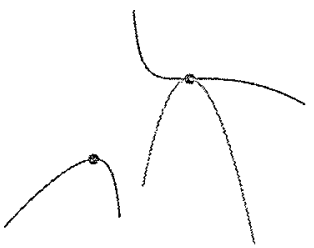

a) Unperturbed, $\delta=-1$.

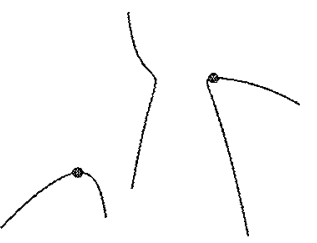

c) Perturbed, $\delta=-1$.

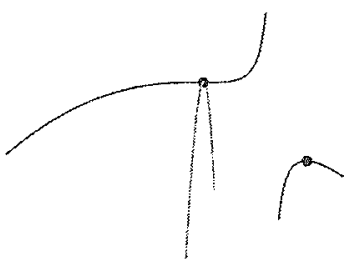

b) Unperturbed, $\delta=1$.

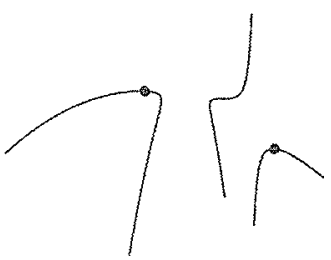

d) Perturbed, $\delta=1$.
Figure 6. Critical paths of the $D_{5}^{ \pm}$-catastrophe in the $(x, y ; t)$-space.

where $\delta= \pm 1$ and $t$ takes the role of $\lambda_{4}$. Its critical curves and catastrophes follow from

$$
\left\{\begin{aligned}
\partial_{x} P U & =\frac{1}{6} x^{3}+x t+\delta\left(t+\frac{1}{2} y^{2}\right) \\
\partial_{y} P U & =\delta x y \\
\operatorname{det}\left(H_{P U}\right) & =\delta x\left(\frac{1}{2} x^{2}+t\right)-y^{2}
\end{aligned}\right.
$$

So the catastrophe points are located at the origin (a double point) and at $(x, y ; t)=\left(-\frac{3}{2} \delta, 0 ;-\frac{9}{8} \delta^{2}\right)$. The latter is a simple annihilation (a fold catastrophe), the former is a cusp catastrophe (three critical points change into one) for both values of $\delta$, see Fig. 6(a) and (b). Indeed the geometry of the $D_{5}$ and its dual are not significantly different. Adding small perturbations by choosing the parameters $\lambda_{1}, \lambda_{2}$, and $\lambda_{3}$, the morsified Cusp catastrophe remains, see Fig. 6(c) and (d). The critical curves at the Cusp breaks up into two curves, one with a Fold catastrophe, one without a catastrophe.

\section{5. $D_{6}^{ \pm}$Second Umbilic Catastrophes}

Ignoring the perturbation terms $\lambda_{1}, \ldots, \lambda_{4}$ for the moment, the scale space expression of the $D_{6}^{ \pm}$catastrophes are given by

$$
\begin{aligned}
S U(x, y ; t)= & \frac{1}{5 !} x^{5}+\frac{1}{3 !} x^{3} t+\frac{1}{2 !} x t^{2} \\
& +\delta\left(\frac{1}{2} x y^{2}+x t\right),
\end{aligned}
$$




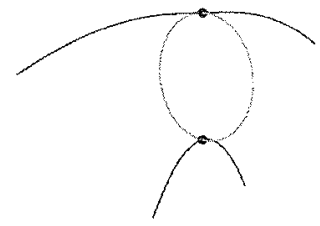

a) Unperturbed, $\delta=1$.

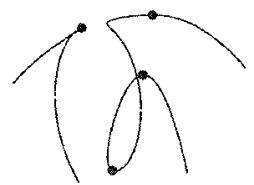

c) Perturbed, $\delta=1$

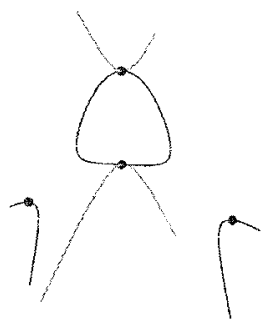

b) Unperturbed, $\delta=-1$

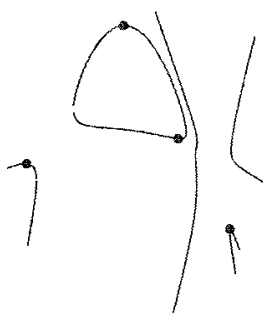

d) Perturbed, $\delta=-1$.
Figure 7. Critical paths of the $D_{6}^{ \pm}$-catastrophe in the $(x, y ; t)$-space.

where $t$ takes the role of $\lambda_{5}$ and $\delta= \pm 1$. Its critical curves and catastrophes follow from

$$
\left\{\begin{aligned}
\partial_{x} S U & =\frac{1}{4 !} x^{4}+\frac{1}{2} x^{2} t+\frac{1}{2} t^{2}+\delta\left(t+\frac{1}{2} y^{2}\right) \\
\partial_{y} S U & =\delta x y \\
\operatorname{det}\left(H_{S U}\right) & =\frac{1}{6} \delta x^{2}\left(x^{2}+6 t\right)-y^{2}
\end{aligned}\right.
$$

Setting $y=0$, several catastrophes occur: If $\delta=1$ there is a scatter event $\left(D_{4}^{-}\right)$at $(x, y ; t)=(0,0 ;-2 \delta)$ and a double Fold event $\left(D_{4}^{+}\right)$at the origin, see Fig. 7(a). If $\delta=-1$, two Fold annihilations occur at $(x, y ; t)=$ $( \pm \sqrt{-6 \delta}, 0 ; \delta)$, and two scatter events at $(x, y ; t)=$ $(0,0 ;-2 \delta)$ and at the origin, see Fig. 7(b).

So the morsification by $t$ of the $D_{6}^{+}$yields a $D_{4}^{-}$scatter followed (while increasing scale) by a $D_{4}^{+}$double annihilation at the origin. The $D_{6}^{-}$shows a $D_{4}^{-}$scatter at the origin, followed by again a $D_{4}^{-}$scatter at some higher scale. Both images show that a part of the critical curve forms a loop: The created critical points annihilate with each other.

If the perturbations are small (or if the measurement contains some uncertainty), one might not be able to distinguish between the involved Fold catastrophes. However, the scale space representation causes a separation into two non-generic catastrophes already men- tioned. Further morsification gives more insight in the way critical curves can behave.

By taking $\lambda_{1}, \ldots, \lambda_{4} \neq 0$, the generic critical curves shown in Fig. 7(c) and (d) are obtained. The morsification of the $D_{6}^{+}$shows two critical curves behaving in an aesthetic way, combining the morsifications of the $D_{4}^{ \pm}$ catastrophes, i.e. containing Fold annihilations and creations. Both created critical points on the right critical curve in Fig. 7(c) annihilate at some larger scale.

The morsification of the $D_{6}^{-}$, on the other hand, still shows the loop close to the origin. Consequently, in contrast to the elliptic Umbilic catastrophe, now both created branches annihilate with each other: the critical curve in the centre of Fig. 7(d) is a closed loop in scale space.

3.5.1. On Generic Loops. The perturbation term of the second Umbilic catastrophes in scale space is given by

$$
\lambda_{1} x+\lambda_{2} y+\lambda_{3}\left(x^{2}+2 t\right)+\lambda_{4}\left(y^{2}+2 t\right) .
$$

If we assume that the image contains the creation catastrophe at the origin, we may set $\lambda_{1}=\lambda_{2}=\lambda_{3}=0$. Furthermore, if it contains an elongated structure at the creation, $\left|\lambda_{4}\right|>0$. This perturbation causes the nongeneric scatter event, visible in Fig. 7(b), to break up. While increasing $\left|\lambda_{4}\right|$ the two parabolae in the $x=0$ plane move along the critical loop, until they meet each other and change into two critical curves without a catastrophe, as shown in Fig. 7(d).

Setting $\lambda_{4}=2 L_{y y}$ we get

$$
\begin{aligned}
S U(x, y ; t)= & L_{x t t}\left(\frac{1}{5 !} x^{5}+\frac{1}{6} x^{3} t+\frac{1}{2} x t^{2}\right) \\
& +L_{x} t\left(x t+\frac{1}{2} x y^{2}\right)+L_{y y}\left(\frac{1}{2} x^{2}+t\right),
\end{aligned}
$$

where $L_{x t t}=L_{x x x x x}$ and $L_{x t}=L_{x y y}$ in absence of other third and fifth order derivatives. Consequently,

$$
\left\{\begin{aligned}
\partial_{x} S U= & L_{x t t}\left(\frac{1}{4 !} x^{4}+\frac{1}{2} x^{2} t+\frac{1}{2} t^{2}\right) \\
& +L_{x t}\left(t+\frac{1}{2} y^{2}\right) \\
\partial_{y} S U= & L_{x t} x y+L_{y y} y \\
\operatorname{det}\left(H_{S U}\right)= & L_{x t t}\left(\frac{1}{6} x^{3}+x t\right)\left(L_{x t} x+L_{y y}\right)-L_{x t} y^{2}
\end{aligned}\right.
$$

One can verify that the solution $x=-\frac{L_{y y}}{L_{x t}}$ of $\partial_{y} S U=0$ describes the non-genericity on the loop if $L_{y y}^{2} \leq$ $(-3+\sqrt{3}) \frac{L_{x t}^{3}}{L_{x t t}}$ by solving $\partial_{x} S U=0$, so $L_{y y}$ needs 
to be large enough and $L_{x t t} L_{x t}<0$, i.e. only the morsified $D_{6}^{-}$pertains its loop. Now at the origin the creation takes place. Note that there $\partial_{t} S U \neq 0$. The successive annihilation follows from $x=y=0$ and $\partial_{x} S U=L_{x t t} \frac{1}{2} t^{2}+L_{x t} t=0$ and takes place at $(x, y ; t)=\left(0,0 ;-\frac{2 L_{x t}}{L_{x t}}\right)$. So the lifetime of the scale space loop is $t_{l}=-\frac{2 L_{x t}}{L_{x t t}}$. Furthermore, the difference in intensity between creation and annihilation yields $L_{y y} t_{l}$. The loop can therefore pertain over a certain range of scales. We will come back to this parameter setting and coordinate choice in Section 4.

\subsection{Morsification Summary}

All non-Fold catastrophes morsify to Fold catastrophes and Morse critical points. The morsification gives insight in the structure around the catastrophe point regarding the critical curves.

The morsification of the Umbilic catastrophes (the $D_{k}$ ) show that the trajectories in scale space of the created critical points fall into several classes.

The morsified $D_{4}^{+}$-catastrophe describes two Fold annihilations. The morsified $D_{4}^{-}$catastrophe describes the creation of a pair of critical points and the annihilation of one of them with another critical point. So while tracing a critical branch of a critical curve both an annihilation and a creation event are traversed.

The morsified $D_{6}^{+}$catastrophe describes the creation of a pair of critical points and the annihilation of both of them with two other critical points. So while tracing a critical branch of a critical curve successively an annihilation, a creation and again an annihilation event are traversed.

The morsified $D_{6}^{-}$-catastrophe describes an isolated closed critical curve, appearing ex nihilo with two critical branches that disappear at some larger scale.

So the morsified $D_{4}^{-}$(and its extension, the $D_{6}^{+}$) and $D_{6}^{-}$-catastrophes describe essentially different creation events in conjunction with the behaviour of the critical curve. An important result lays on the area of tracing critical points. If one traces only critical points starting

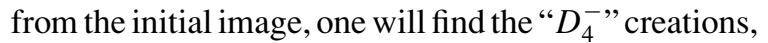
since they emerge as the starting point of a part of a critical curve that annihilates with one of the initial critical points. However, one will miss the " $D_{6}^{-}$" loops that occur somewhere in scale space, since they have no relation to the critical points in the initial image by means of the critical curves emerging from these points. So fine-to-coarse tracing of critical points will not always yield all critial curves.
Note that the full morsification of the non-generic catastrophes always yields the generic Fold annihilations and creations and Morse critical points.

3.6.1. Other Non-Generic Catastrophes. The catastrophes, their scale space formulation, and their morsifications, as treated in the previous sections, are only the beginning of an infinite set of possible descriptions on the behaviour of critical curves. Due to extreme local symmetries and inaccuracies - think of a large checkerboard pattern-one might encounter nongeneric events to be described with higher other catastrophe models. These models follow in a straightforward manner from the route we described.

From the point of view of distinct appearances of critical curves, this expose suffices. Since the only possible generic catastrophes on critical curves are creations and annihilations of pairs of critical points, the possibilities are limited. Given a critical curve, it either originated from the initial image and the curve can contain several protuberances, i.e. sequences of successive creations and annihilations, or it didn't originate from the initial image and it forms a loop, perhaps also with protuberances. Both possibilities are described by the aforementioned catastrophe models. The scale space formulations of the "three exceptional catastrophes" don't yield any extra information, as one can verify.

\section{Applications}

In this section we give some examples to illustrate the theory presented in the previous sections. We will focus on the critical curves containing a creation event. An example of desired symmetry in an image and consequently modelling by a Cusp catastrophe has been presented elsewhere by the authors Kuijper and Florack (2001b). Firstly, appearance of the morsified $D_{4}^{-}$-catastrophe on critical paths is shown using the artificial MR image of Fig. 8(a). This image is taken from the "Brain Web". 4 Secondly, an example of creation ex nihilo, the morsified $D_{6}^{-}$-catastrophe, is shown by means of the classic "bridge"-image of Fig. 11(a), and the aformentioned MR image.

\section{1. $D_{4}^{-}$Catastrophe}

The artificial MR image of Fig. 8(a) was used as initial image for the scale space image. For visualisation purposes, we restricted to the scale range 8.37-33.1. 


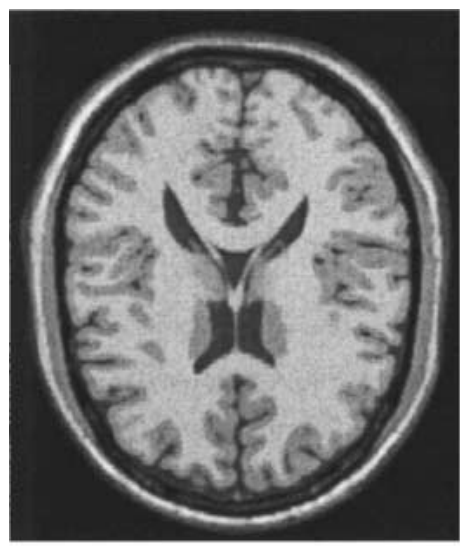

a)

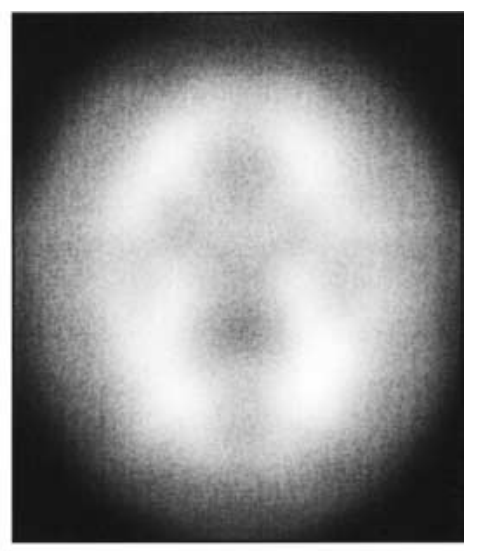

b)

Figure 8. (a) $181 \times 217$ artificial MR image. (b) Image on scale 8.37.

The image at scale 8.37 (with only the large structures remaining) is shown in Fig. 8(b). This image contains 7 extrema.

The scale space image in this scale range contains 161 logarithmically sampled scales. At all scales the spatial critical points were calculated and connected, forming the critical paths. Figure 9 shows these critical paths in the $(x, y ; t)$-space. The bright curves represent the extrema, the dark ones the saddles. At the (approximate) catastrophe locations the curves are connected. Globally, the image shows annihilating pairs of critical points. Locally, however, the presence of extra branches of critical curves is visible.

A close-up of one of the critical paths is shown in Fig. 10(a). It clearly shows a critical curve containing two subsequent Fold annihilation — creation events. The critical curve evidently shows the appearance of an annihilation-creation pair described by the $D_{4}^{-}$morsification. Note that the creation events would have been missed if the sampling was taking coarser, yielding one critical curve without protuberances in scale direction. Sampling without connecting critical paths yields the observation of temporarily created extrema (and saddles), cf. Simmons et al. (1998).

\section{2. $D_{6}^{-}$Catastrophe}

Figure 11(a) shows the classical "bridge"-image: two mountains of different height (blobs with different intensity) connected by a small ramp and a deep valley between the mountains. This image was described by Lifshitz and Pizer (1990) as possible initial image yielding a creation event in scale space.

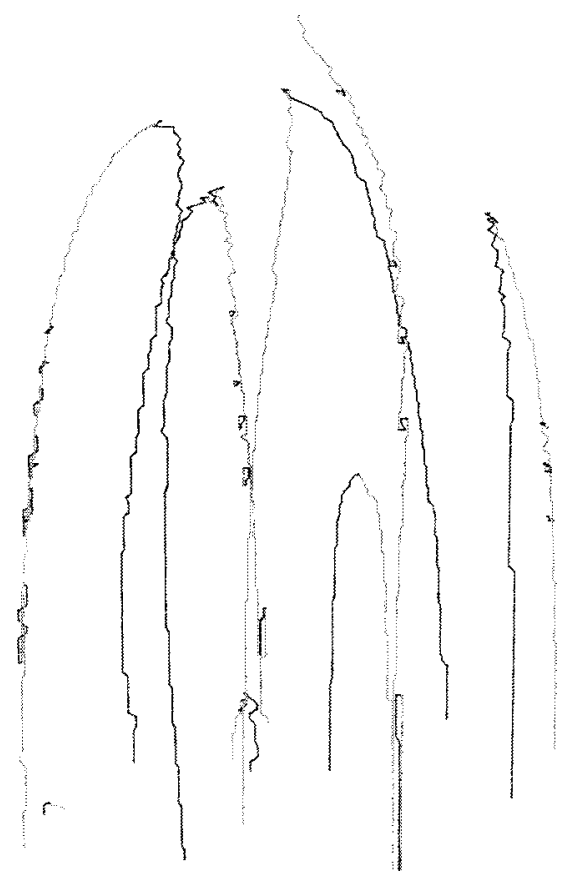

Figure 9. Critical paths (bright part represent the extrema, the dark parts the saddles) of the MR image in scale range 8.37-33.1.

- Firstly, there is only one maximum of the left blob. The right blob is not a maximum, since it is connected to the other blob by the ramp.

- Secondly, at some scale the ramp changes into a bridge with a deep dip in it due to the surrounding deep valleys: a maximum (right blob)—saddle (dip of the bridge) pair is created.

- Finally, at a large scale a saddle-extremum annihilation occurs. 


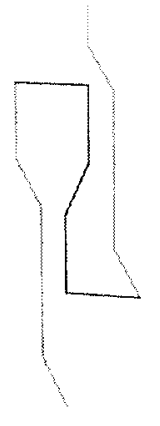

a)

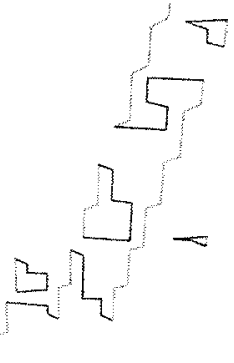

b)
Figure 10. (a) Close-up of one of the critical paths of the MR image, showing a subsequent annihilation-creation event. (b) Close-up, showing subsequent annihilation-creation events and loop events.

If the saddle annihilates with the left extremum, it can be modelled by the $D_{4}^{-}$catastrophe, as in the previous section. However, as shown by Fig. 11(b), it can also annihilate with the newly created extremum. This figure shows the critical paths of the scale space image of Fig. 11(a). The left string represents the extremum of the brightest blob, the loop represents the created and annihilated maximum-saddle pair.

Since the structure is built up as discussed in Section 3.5.1, the loop remains over a relatively large number of scales. Due to mirror symmetry we know that the $x$-direction is the one indeed corresponding to that used in the previous canonical models. Numerical calculations show a very strong response on the fraction $\frac{L_{x y y}}{L_{x x x x x}}$ close to the scale space location of the creation, as predicted by the model as measure for the lifetime of the loop.

The same behaviour is observed at the MR scale space image. Figure 10(b) shows a close-up of one of the critical curves. Besides several aforementioned subsequent Fold annihilation-creation events along the critical curve, here clearly also several "loop events" occur.

\section{Discussion}

In this paper we investigated the (deep) structure on various catastrophe events in Gaussian scale space. Although it is known that pairs of critical points are annihilated and created (the latter if the dimension of the image is 2 or higher), it is important to describe also the local structure of the image around the non-generic events. These events might be encountered in practical usage of scale spaces and the non-generic catastrophes can be used to model these occurrences. We therefore embedded catastrophes in scale space. Scale acts as one of the perturbation parameters. The morsification of the catastrophes yields generic Fold annihilations and creations of pairs of critical points.

The $A_{k}$ series can be used to model (almost) simultaneous annihilations of pairs of critical points at a location (or indistinguishable region) in scale space. If $k$ is even, it models the annihilation of $k$ critical points, if $k$ is odd, it models the collision of $k$ critical points where $k-1$ annihilate and one remains.

For creations the $D_{k}$ series can be used. Creations occur in different types. Critical paths in scale space can have protuberances, a subsequent occurrence of an annihilation and a creation. In scale space images this is visible by the creation of an extremum-saddle pair, of which one critical point annihilates at some higher scale with an already present critical point, while the other remains unaffected. It is also possible that critical paths form loops: the created pair annihilates at some

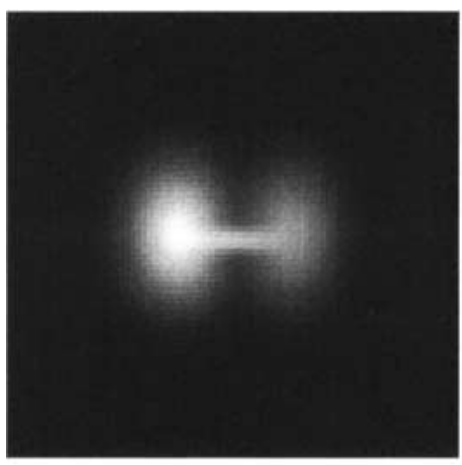

a)

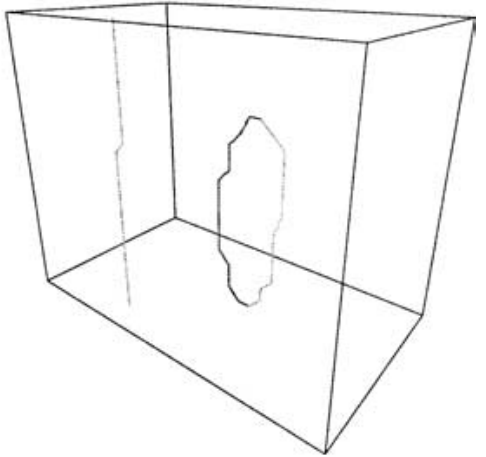

b)

Figure 11. (a) Artificial bridge image. (b) Critical paths of the bridge image. 
higher scale. The possibility for both types to occur in practice was shown in the artificial MR image. This phenomena is known from physics, where it is used to describe the creation and successive annihilations of "virtual" elementary particles (and even the universe) (e.g., Argyris et al., 2001; Maslov and Shagalov, 2001; Moss, 2000).

Furthermore we showed that the protuberances in the critical paths, expressed in canonical coordinates, occur only in case of a small local perturbation. In addition, creations are less likely to happen due to a special constraint on the combination of third order derivatives and local perturbation. We gave a dimension dependent expectation of this event and an upper bound for the perturbation in canonical coordinates. An different approach, based on the 2D image coordinate system, was taken by the authors in Kuijper and Florack (1999) and Florack and Kuijper (2000). It led to the definition of a so-called "potential creation area", based on the third order derivatives. This area varied, depending on the type of image and the scale, between 0.04 and 0.12 .

The lifetime of a created pair is enlarged if the local structure is elongated. This was derived from the canonical formulation and visualised by the example of the bridge image in Section 4.

Since the number of possible catastrophes is infinite, there is an infinite number of possible non-generic constellations in which ("infinite") critical points are annihilated and created. We restricted ourselves to the situations in which at most 6 critical points annihilate and in which critical points are created, the latter divided into models representing protuberances and loops.

Finally, the calculations were based on the canonical coordinates. In general, it is not trivial to transform the local coordinate system to these nice formulated catastrophe germs. In that sense, the numerical values have no direct meaning. They do describe, however, the qualitative behaviour of the critical curves close to the location of the catastrophes and can therefore be used to model the type of behaviour encountered in practical usage of a scale space. We gave examples of the appearances of this behaviour in Section 4 based on an artificial MR image.

The theory described in this paper extends the knowledge of the deep structure of Gaussian scale space, especially with respect to the behaviour of critical curves in the vicinity of creation events and the scale space lifetime of the created critical points. It emphasises the relevance of investigating the complete scale space image, instead of a series of images at different scales.

\section{Acknowledgments}

James Damon and Steve Pizer of the University of North Carolina are gratefully acknowledged for their comments on a preliminary version of this paper.

\section{Notes}

1. This can be modelled by a large perturbation of $\lambda_{2}$ in Eq. (7). What happens in practice is a violation of the constraint that the local model descriptions of the subsequent Fold catastrophes are only valid in a small environment of the catastrophe.

2. Of course, morsification of more complicated catastrophes can also give birth to a creation event. If the critical curves behave similar, the morsified scale space elliptic Umbilic catastrophe is the simplest when taking into account the number of perturbation parameters. However, they may also describe different behaviour of the critical curve, see Section 3.5.1.

3. In general, it is not trivial to transform the local coordinate system to these nice formulated catastrophe germs. In that sense, the numerical values have no direct meaning. They do describe, however, the qualitative behaviour of the critical curves close to the location of the catastrophes and can therefore be used to model the type of behaviour encountered in practical usage of a scale space.

4. http://www.bic.mni.mcgill.ca/brainweb, see e.g. Cocosco et al. (1997).

\section{References}

Argyris, J., Ciubotariu, C., and Matuttis, H.G. 2001. Fractal space, cosmic strings and spontaneous symmetry breaking. Chaos, Solitons and Fractals, 12(1):1-48.

Arnold, V.I. (Ed.). 1983. Geometrical Methods in the Theory of Ordinary Differential Equations, vol. 250 of Grundlehren der mathematischen Wissenschaften: A Series of Comprehensive Studies in Mathematics. Springer-Verlag: Berlin.

Arnold, V.I. 1984. Catastrophe Theory. Springer: Berlin.

Arnold, V.I. (Ed.). 1992. Ordinary Differential Equations. SpringerVerlag: Berlin.

Arnold, V.I. (Ed.). 1993a. Dynamical Systems VI: Singularity Theory I, vol. 6 of Encyclopaedia of Mathematical Sciences. Springer-Verlag: Berlin.

Arnold, V.I. (Ed.). 1993b. Dynamical Systems VIII: Singularity Theory II \& Applications, vol. 39 of Encyclopaedia of Mathematical Sciences. Springer-Verlag: Berlin.

Arnold, V.I. (Ed.). 1994. Dynamical Systems V: Bifurcation Theory and Catastrophe Theory, vol. 5 of Encyclopaedia of Mathematical Sciences. Springer-Verlag: Berlin.

Berkner, K. 1999. Resolution of singularities of convolutions with the Gaussian kernel. Proc. Amer. Math. Soc., 127(2):409415.

Bruce, J.W. and Giblin, P.J. 1984. Curves and Singularities. Cambridge University Press.

Clark, J.J. 1988. Singularity theory and phantom edges in scale space.

Cocosco, C.A., Kollokian, V., Kwan, R.K.-S., and Evans, A.C. 1997. BrainWeb: Online interface to a 3D MRI simulated brain 
database. NeuroImage, 5(4, part 2/4):S425. In Proceedings of 3-rd International Conference on Functional Mapping of the Human Brain, Copenhagen, May 1997.

Damon, J. 1995. Local Morse theory for solutions to the heat equation and Gaussian blurring. Journal of Differential Equations, 115(2):386-401.

Damon, J. 1997a. Generic properties of solutions to partial differential equations. Arch. Rat. Mech. Anal., 353-403.

Damon, J. 1997b. Local Morse theory for Gaussian blurred functions. Gaussian Scale-Space Theory, J. Sporring, M. Nielsen, L.M.J. Florack, and P. Johansen (Eds.), vol. 8 of Computational Imaging and Vision Series, 2nd edn. Kluwer Academic Publishers: Dordrecht, pp. 147-162.

Duncan, J. and Ayache, N. 2000. Medical image analysis: Progress over two decades and the challenges ahead. IEEE Transactions on Pattern Analysis and Machine Intelligence, 22(1):85-105.

Eberly, D., Gardner, R., Morse, B., Pizer, S., and Scharlach, C. 1994. Ridges for image analysis. Journal of Mathematical Imaging and Vision, 4(4):353-373.

Florack, L.M.J. 1997. Image Structure, vol. 10 of Computational Imaging and Vision Series. Kluwer Academic Publishers: Dordrecht, The Netherlands.

Florack, L.M.J. and Kuijper, A. 2000. The topological structure of scale-space images. Journal of Mathematical Imaging and Vision, 12(1):65-80.

Florack, L.M.J., ter Haar Romeny, B.M., Koenderink, J.J., and Viergever, M.A. 1992. Scale and the differential structure of images. Image and Vision Computing, 10(6):376-388.

Florack, L.M.J., ter Haar Romeny, B.M., Koenderink, J.J., and Viergever, M.A. 1993. Cartesian differential invariants in scalespace. Journal of Mathematical Imaging and Vision, 3(4):327348

Florack, L.M.J., ter Haar Romeny, B.M., Koenderink, J.J., and Viergever, M.A. 1994. General intensity transformations and differential invariants. Journal of Mathematical Imaging and Vision, 4(2): 171-187.

Florack, L.M.J., ter Haar Romeny, B.M., Koenderink, J.J., and Viergever, M.A. 1994. Linear scale-space. Journal of Mathematical Imaging and Vision, 4(4):325-351.

Florack, L.M.J., ter Haar Romeny, B.M., Koenderink, J.J., and Viergever, M.A. 1996. The Gaussian scale-space paradigm and the multiscale local jet. International Journal of Computer Vision, 18(1):61-75.

Fomenko, A.T. and Kunii, T.L. 1997. Topological Modeling for Visualization. Springer-Verlag: Tokyo.

Gilmore, R. 1993. Catastrophe Theory for Scientists and Engineers. Dover. Originally published by John Wiley \& Sons: New York (1981).

Griffin, L.D. 1997. Critical point events in affine scale-space Gaussian Scale-Space Theory, J. Sporring, M. Nielsen, L.M.J. Florack, and P. Johansen (Eds.), vol. 8 of Computational Imaging and Vision Series, 2nd edn. Kluwer Academic Publishers: Dordrecht, pp. 165-180.

Griffin, L.D. and Colchester, A. 1995. Superficial and deep structure in linear diffusion scale space: Isophotes, critical points and separatrices. Image and Vision Computing, 13(7):543557.

Griffin, L.D., Colchester, A., and Robinson, G. 1992. Scale and segmentation of grey-level images using maximum gradient paths. Image and Vision Computing, 10(5):389-402.
Henkel, R.D. 1995. Segmentation in scale space. In Computer Analysis of Images and Patterns. Lecture Notes in Computer Science, vol. 970. Springer-Verlag, pp. 41-48.

Iijima, T. 1962. Basic theory of pattern normalization (for the case of a typical onedimensional pattern). Bulletin of the Electrotechnical Laboratory, 26:368-388 (in Japanese).

Johansen, P. 1994. On the classification of toppoints in scale space. Mathematical Imaging and Vision, 4(1):57-67.

Johansen, P. (Ed.). 1996. Proceedings of the copenhagen workshop on Gaussian scale-space theory, May 10-13. Technical Rapport 96/19 ISSN 01078283.

Johansen, P. 1997. Local analysis of image scale space. Gaussian Scale-Space Theory, J. Sporring, M. Nielsen, L.M.J. Florack, and P. Johansen (Eds.), vol. 8 of Computational Imaging and Vision Series, 2nd edn. Kluwer Academic Publishers: Dordrecht, pp. 139-146.

Johansen, P. 1997. Local analysis of scale space. Technical Report 97/2, Department of Computer Science, University of Copenhagen.

Johansen, P., Nielsen, M., and Olsen, O.F. 2000. Branch points in one-dimensional Gaussian scale space. Journal of Mathematical Imaging and Vision, 13:193-203.

Johansen, P., Skelboe, S., Grue, K., and Andersen, J.D. 1986. Representing signals by their toppoints in scale space. In Proceedings of the International Conference on Image Analysis and Pattern Recognition (Paris, France, October 1986), IEEE Computer Society Press, pp. 215-217.

Kalitzin, S.N. 1997. Topological numbers and singularities. Gaussian Scale-Space Theory, J. Sporring, M. Nielsen, L.M.J. Florack, and P. Johansen (Eds.), vol. 8 of Computational Imaging and Vision Series, 2nd edn. Kluwer Academic Publishers: Dordrecht, pp. 181-190.

Kalitzin, S.N., ter Haar Romeny, B.M., Salden, A.H., Nacken, P.F.M., and Viergever, M.A. 1998. Topological numbers and singularities in scalar images. Scale-space evolution properties. Journal of Mathematical Imaging and Vision, 9(3):253-296.

Kalitzin, S.N., Staal, J., ter Haar Romeny, B.M., and Viergever, M.A. 2001. A computational method for segmenting topological point-sets and application to image analysis. IEEE Transactions on Pattern Analysis and Machine Intelligence, 23(5):447-459.

Keller, R.S. 1999. Generic transitions of relative critical sets in parametrized families with applications to image analysis. Ph.D. Thesis, University of North Carolina.

Kerckhove, M. (Ed.). 2001. Scale-Space and Morphology in Computer Vision, vol. 2106 of Lecture Notes in Computer Science. Springer-Verlag: Berlin Heidelberg.

Koenderink, J.J. 1984. The structure of images. Biological Cybernetics, 50:363-370.

Kuijper, A. and Florack, L.M.J. 1999. Calculations on critical points under gaussian blurring. Scale-Space Theories in Computer Vision, M. Nielsen, P. Johansen, O.F. Olsen, and J. Weickert (Eds.), vol. 1682 of Lecture Notes in Computer Science. Springer-Verlag: Berlin Heidelberg, pp. 318-329.

Kuijper, A. and Florack, L.M.J. 2001a. Hierarchical presegmentation without prior knowledge. In Proceedings of the 8th International Conference on Computer Vision (Vancouver, Canada, July 9-12, 2001), pp. 487-493.

Kuijper, A. and Florack, L.M.J. 2001b. The application of catastrophe theory to image analysis. Technical Report UU-CS-2001-23, Department of Computer Science, Utrecht University (submitted). 
Kuijper, A. and Florack, L.M.J. 2002. Logical filtering in scale space. Technical Report UU-CS-2002-018, Department of Computer Science, Utrecht University, Accepted for publication as "The hierarchical structure of images" in IEEE Transactions on Image Processing.

Kuijper, A., Florack, L.M.J., and Viergever, M.A. 2003. Scale space hierarchy. Journal of Mathematical Imaging and Vision, 18(2):169-189.

Lifshitz, L.M. and Pizer, S.M. 1990. A multiresolution hierarchical approach to image segmentation based on intensity extrema. IEEE Transactions on Pattern Analysis and Machine Intelligence, 12(6):529-540.

Lindeberg, T. 1992. Scale-space behaviour of local extrema and blobs. Journal of Mathematical Imaging and Vision, 1(1):65-99.

Lindeberg, T. 1993. Detecting salient blob-like image structures and their scales with a scale-space primal sketch: A method for focus-of-attention. International Journal of Computer Vision, 11(3):283-318.

Lindeberg, T. 1994. Scale-Space Theory in Computer Vision. The Kluwer International Series in Engineering and Computer Science. Kluwer Academic Publishers.

Lu, Y.-C. 1976. Singularity Theory and an Introduction to Catastrophe Theory. Springer-Verlag: Berlin, second corrected printing edition.

Maslov, E.M. and Shagalov, A.G. 2001. Dynamics of first-order phase transitions in the $\phi^{4}-\phi^{6}$ model caused by the parametric instability of the metastable vacuum. Physica $D$ : Nonlinear Phenomena, 152/153:769-778.

Moss, I.G. 2000. Heat kernel expansions for distributional backgrounds. Physics Letters B, 491(1/2):203-206.

Nielsen, M., Johansen, P., Olsen, O.F., and Weickert, J. (Eds.). 1999. Scale-Space Theories in Computer Vision, vol. 1682 of Lecture Notes in Computer Science. Springer-Verlag: Berlin Heidelberg.

Olsen, O.F. 1997. Multi-scale watershed segmentation. Gaussian Scale-Space Theory, J. Sporring, M. Nielsen, L.M.J. Florack, and P. Johansen (Eds.), vol. 8 of Computational Imaging and Vision Series, 2nd edn. Kluwer Academic Publishers: Dordrecht, pp. 191200.

Olsen, O.F. 2000. Generic image structure. Ph.D. Thesis, University of Copenhagen, Denmark.

Olsen, O.F. and Nielsen, M. 1997. Generic events for the gradient squared with application to multi-scale segmentation. Scale-Space Theory in Computer Vision: Proceedings of the First International Conference, Scale-Space' 97, B.M. ter Haar Romeny, L.M.J. Florack, J.J. Koenderink, and M.A. Viergever, (Eds.), Utrecht, The Netherlands, vol. 1252 of Lecture Notes in Computer Science. Springer-Verlag: Berlin, pp. 101-112.

Otsu, N. 1981. Mathematical studies on feature extraction in pattern recognition. Ph.D. Thesis, Electronical Laboratory, Ibaraki, Japan (in Japanese).

Poston, T. and Stewart, I.N. 1978. Catastrophe Theory and its Applications. Pitman: London.
Rieger, J.H. 1992. Generic properties of edges and corners on smooth greyvalue surfaces. Biological Cybernetics, 66:497502.

Rieger, J.H. 1995. Generic evolutions of edges on families of diffused greyvalue surfaces. Journal of Mathematical Imaging and Vision, 5:207-217.

Rieger, J.H. 1997. Topographical properties of generic images. International Journal of Computer Vision, 23(1):79-92.

Salden, A.H. 1996. Dynamic scale-space paradigms. Ph.D. Thesis, Utrecht University.

Simmons, A., Arridge, S.R., Tofts, P.S., and Barker, G.J. 1998. Application of the extremum stack to neurological MRI. IEEE Transactions on Medical Imaging, 17(3):371-382.

Sporring, J., Nielsen, M., Florack, L.M.J., and Johansen, P. (Eds.). 1997. Gaussian Scale-Space Theory, vol. 8 of Computational Imaging and Vision Series, 2nd edn. Kluwer Academic Publishers: Dordrecht.

ter Haar Romeny, B.M., Florack, L.M.J., Koenderink, J.J., and Viergever, M.A. (Eds.). 1997. Scale-Space Theory in Computer Vision: Proceedings of the First International Conference, ScaleSpace' 97, Utrecht, The Netherlands, vol. 1252 of Lecture Notes in Computer Science. Springer-Verlag: Berlin.

Thom, R. 1972. Stabilité Structurelle et Morphogénèse. Benjamin: New York.

Thom, R. 1975. Structural Stability and Morphogenesis. BenjaminAddison Wesley (translated by D.H. Fowler).

Wada, T. and Sato, M. 1990. Scale-space tree and its hierarchy. In ICPR90, vol. II, pp. 103-108.

Weickert, J.A., Ishikawa, S., and Imiya, A. 1997. On the history of Gaussian scale-space axiomatics. Gaussian Scale-Space Theory, J. Sporring, M. Nielsen, L.M.J. Florack, and P. Johansen (Eds.), vol. 8 of Computational Imaging and Vision Series, 2nd edn. Kluwer Academic Publishers: Dordrecht, pp. 45-59.

Weickert, J.A., Ishikawa, S., and Imiya, A. 1999. Linear scale-space has first been proposen in Japan. Journal of Mathematical Imaging and Vision, 10(3):237-252.

Witkin, A.P. 1983. Scale-space filtering. In Proceedings of the Eighth International Joint Conference on Artificial Intelligence, pp. 1019-1022.

Woodcock, A.E.R. and Davis, M. (Eds.). 1978. Catastrophe Theory. Clarke, Irwin \& Company Limited.

Zeeman, E.C. 1977. Catastrophe Theory: Selected Papers, 1972 1977. Addison-Wesley Publishing Company.

Zhao, N. 1985. Feature extraction of images by stable gaze tree. Ph.D. Thesis, Department of Cumputer Science, Tokyo Institute of Technology, Japan (in Japanese).

Zhao, N. and Iijima, T. 1985a. A theory of feature extraction by the tree of stable view-points. IECE Japan, Trans. D, J68-D:11251132 (in Japanese).

Zhao, N. and Iijima, T. 1985b. Theory on the method of determination of view-point and field of vision during observation and measurement of figure. IECE Japan, Trans. D, J68-D:508-514 (in Japanese). 